Künftigen Forschern bleibt es vorbehalten, die für die Biologie höchst wichtige Frage zu lösen, ob die neuentstandene Bastardform schliefslich vollkommen in ihren Stammarten aufgeht oder umgekehrt sich als Ursprung einer neuen Art erweist, die mit der Zeit konstante Kennzeichen erwirbt und eine der wichtigsten Fragen der Biologie löst, indem sie einen der Wege zur Bildung in der Natur neuer Arten des Tierreichs feststellt. Die Frage ist jedenfalls von so grofser Bedeutung für die gesammte biologische Wissenschaft, dafs alle sich für biologische Fragen interessierenden Personen und Körperschaften ihr ihre vollste Aufmerksamkeit zollen sollten.

\title{
Bericht über die 60. Jahresversammlung der Deutschen Ornithologischen Gesellschaft in Eberswalde und Freienwalde rom 7. bis 9. Oktober 1911.
}

Anwesend die Herren: Deditius (Berlin), Schalow (Berlin), Re ic he n ow (Berlin), J ung (Berlin), Voig t (Leipzig), Hesse (Berlin), Kra a se (Berlin), Graf v. Zed lit z und Trü tzschler (Schwentnig), v. Luc an us (Berlin), K. Neunzig (Hermsdorf), $\mathrm{H}$ e i $\mathrm{r}$ o $\mathrm{th}$ (Berlin), D o m e i e r (Uckermünde), O. N e u m an n (Berlin).

Als Gäste die Herren: Hild ebrandt (Altenburg), R. $\mathrm{N}$ e unzig (Hermsdorf), Eckstein (Eberswalde) und Frau $\mathrm{H}$ e i n r o th (Berlin).

Vorsitzender: Herr $\mathrm{S} \mathrm{c}$ h alow.

Schriftführer: Herr $\mathrm{H}$ e in rot $h$.

Am Sonnabend den 7. Oktober abends 8 Uhr, fand der Begrürsungsabend im Hotel "Deutsches Haus" statt, den Herr $\mathrm{Sch}$ a lo $\mathrm{w}$ als Vorsitzender eröffnete.

Die Herren Tischler (Heilsberg), v. T resk ow (Berlin), Freiherr ve Berlepsch (Seebach), W. B lasi us (Braunschweig), Nehrkorn (Braunschweig) und H el m (Chemnitz) haben telegraphische oder schriftliche Grüfse übersandt und ihrem Bedauern Ausdruck gegeben, an den Sitzungen der Jahresversammlung nicht teilnehmen zu können.

Herr D e d iti us gibt den Kassenbericht über die letzten 3 Jahre. Herr S c hal ow dankt dem Kassenführer für seine hingebenden Mühewaltungen im Interesse der Gesellschaft. Die Herren Vo i $\mathrm{t}$ und $\mathrm{H}$ e s s e werden mit der Prüfung der Rechnung beauftragt.

Bei der Besprechung der Tagesordnung für die kommenden Tage wird auf Vorschlag des Herrn E cks t e i n beschlossen, sich am Sonntag früh nach der Moltke-Treppe am,Rande des Waldes zu begeben, da dort häufig um diese Jahreszeit ein interessanter und ausgedehnter Krähenzug stattzufinden pflegt. Gegen 9 Uhr soll 
dann das Zoologische Museum der Königl. Forstakademie aufgesucht werden und um 1 Uhr im Hôtel Deutsches Haus das gemeinsame Mittagessen stattfinden. Von Nachmittags $4 \mathrm{Uhr}$ $a b$ sind Vorträge in Aussicht genommen. Herr Dr. L a valle in Schiffmühle bei Freienwalde hat in liebenswürdiger Weise die Mitglieder der Jabresversammlung aufgefordert, seine Geflügelzüchterei zu besuchen. Man beschliefst daher, am Montag, den 9. Oktober, vormittags von Eberswalde abzufahren, in NiederFinow die grofsartigen Schleusen und Kanalbauten des SpreeOder Kanals zu besichtigen und dann nach Freienwalde weiterzufahren, daselbst zu Mittag zu essen und nachmittags gegen 3 Uhr Herrn Dr. L a valle aufzusuchen. Die Tagesordnung in der vorgelegten Form wird angenommen.

Herr E c ks t ein legt eine bei Eberswalde geschossene und ihm eingelieferte Ente zur Bestimmung vor. Herr $\mathrm{H}$ e in $\mathrm{r}$ ot h erklärt das Tier für eine Hausente und zwar für einen vicht ganz reinen Vertreter der Smaragdentenrasse. Das 'Tier hat etwa Stockentengröfse und ist im wesentlichen mattschwarz mit einigen weifsen Federn an der Hals- und Kropfgegend. Nach seinen Erfahrungen treibt sich gerade diese leichte und verhältnismäfsig gutfliegende Entenrasse weit umber und wird öfters erlegt.

Herr Reich e n ow wirft die Frage auf, wieweit die im Osten Deutschlands in diesem Jahre bereits häufiger beobachteten Tannenheher schon nach Westen vorgedrungen seien. Am 22. September hat er den ersten Vogel der Art eingeliefert erhalten. Die Herren $G r$ af v. $Z$ e d lit $z$, v. L u c a n u s und $E \mathrm{cks}$ e i n haben diese Art verschiedentlich Ende September in Schlesien, Pommern und der Mark gesehen, Herr R. Neunzig hat 6 Tage lang täglich einige Stücke bei Jüterbogk beobachtet. Auch Herr $\mathrm{Hildeb} \mathbf{r a n d}$ hat in Altenburg schon einige Tannenheher erbalten; es handelt sich dabei stets um den sibirischen, dünnschnäbligen Vogel. Herr R eichenow regt die Frage an, warum wohl ein so starker Tannenheherzug stattfinde, und ob dieser wohl durch Nahrungsmangel in der Heimat der Vögel verursacht werde. Leider fehlt es darüber an jeder Erfahrung.

Herr S $\mathrm{ch}$ a lo w berichtet, dals in den Vereinigten Staaten von Nordamerika seitens der $L$ in $n$ ea $n$ Society of $\mathrm{New}$ Y o r $\mathbf{k}$ das Beringen der Vögel in grolsartigem Malsstabe ausgeführt werden solle; er verspricht sich von diesen Versuchen ganz ausgezeichnete Erfolge für die Kenntnis der Wanderungen nordamerikanischer Vögel.

Herr Ecks te in meldet, dafs sich heute gegen Abend die ersten Saatgänse in Eberswalde eingefunden hätten.

Herr Schalow spricht über den demnächst erscheinenden Bericht über die Verhandlungen des V. Internationalen Ornithologen Kongresses und rechtfertigt das späte Erscheinen desselben. Er schildert die Schwierigkeiten, die er mit einigen Verfassern 
gehabt hat, deren zum Teil ungemein säumige Einlieferung ihrer Arbeiten an der Verzögerung schuld ist.

Herr Voig t erkundigt sich nach dem Verhältnis der Abhängigkeit bezw. der Zugehörigkeit der Vogelwarte Rossitten zu der Deutschen Ornithologiscben Gesellschaft. Herr Re ich e n ow beantwortet die Frage dahin, dals die Vogelwarte der Deutschen Ornithologischen Gesellschaft gehöre, dafs das Museum derselben auf fiskalischem Boden, aus staatlichen Mitteln gebaut sei, und dals sie eine jährliche Unterstützung seitens der Ministerien des Kultus und der Land- und Forstwirtschaft erhält. Aufserdem wird das Gehalt des Herrn Prof. Dr. Thienemann, der der Universität Königsberg als Kustos angegliedert ist, aus staatlichen Mitteln bezahlt, während die Kosten für die Drucksachen, Bericht über die Vogelwarte, etc. die Ornithologische Gesellschaft trägt. Herr Vo ig t dankt für die Aufklärung. Er berichtet auf eine Anfrage des Vorsitzenden über die Lage der Verhältnisse in Hiddensee, bezw. über eine daselbst zu errichtende Schutzstation. Die Insel Hiddensee gehört zu Stralsund, ihr Hauptort ist Kloster. Es sind Meinungsverschiedenheiten $z$ wischen dem „Frauenbund zum Schutze der Vogelwelt", vertreten durch Herrn Steinmetz, und dem Stralsunder Ornithologischen Verein entstanden, die beide die Vogelbrutkolonien auf und um Hiddensee schützen wollen. Namentlich die Insel Fehr und der Gänsewerder kommen hierbei in Betracht. Die praktische Ausübung des Vogelschutzes liegt hauptsächlich in den Händen des sehr interessierten und aufopferungsfreudigen Lehrers Segebrecht in Vitte. Es wird sich darum handeln, diesen Herrn für seine eifrigen Bemühungen in geeigneter Weise zu entschädigen.

Herr S c ha low dankt für den sehr interessanten Bericht. Herr Eckstei n rät, dals man Herrn Geheimrat Professor Dr. Conwentz zur Schlichtung des Streites für die Sache interessieren solle.

Herr V o ig t betont, dals es sich vor allen Dingen darum handle, dals die Jagdscheine, die in Hiddensee an Kurgäste vergeben würden und eine Einnabmequelle des Pächters in Kloster darstellten, abgeschafft werden mülsten, Herr $\mathbf{E} \mathrm{c} \mathrm{k}$ s t e i n glaubt, dafs die Landesverwaltung bezw. Stralsund hierbei einschreiten können.

Zum Schlusse der Abendsitzung wirft Herr E c k s te in die Frage auf, wie es möglich sei, dafs man trotz aller Vogelschutzgesetze in Deutschland z. B. für Magenuntersuchungen beliebig viele Mägen geschützter Vögel erhalten könne. Auch sei es erstaunlich, das man jederzeit alle möglichen Singvögel unter der Hand kaufen kann.

Bei einem Meinungsaustausch hierüber wird darauf hingewiesen, dafs die häufiger im Käfige gehaltenen Körnerfresser in grofsen Mengen aus Holland und Böhmen eingeführt würden. Einzelne Vögel werden natürlich auch in Deutschland unerlaubter 
Weise häufig gefangen. Die Schuld an diesen Gesetzesüberschreitungen trage dabei namentlich derjenige, von dem die Nachfrage nach geschützten Vögeln ausgeht.

Am Sonntag, den 8. Oktober begaben sich die Teilnehmer der Jahresversammlung nach der Moltke-Treppe, wo sich von der Höhe ein schöner Blick über Eberswalde eröffnet. Leider blieben die Krähen aus, denn es war regnerisches, trübes Wetter.

Herr E ckstein geleitete die Versammelten nach seiner Wohnung. Dort tummelten sich im Garten drei reizende, junge Fischottern bei einer Kollihündin, die ihnen als Amme gedient hatte. Es gewährte viel Vergnügen, die munteren Spiele der wolligen kleinen Geschwister $z \mathbf{u}$ beobachten.

Hierauf wurde das Zoologische Museum der Forstakademie besucht. Es enthält eine schöne Sammlung einheimischer 'Tiere, von denen natürlich die Vögel besonders in Augenschein genommen wurden. $\mathrm{Zu}$ zwanglosen Gruppen vereinigt besichtigten die Teilnehmer der Jahresversammlung die interessanten Stücke der Sammlung. Einige Farben-Varietäten fanden besonderen Anklang, von seltenen Irrgasten sei Elanus erwähnt.

Um 1 Uhr fand das Mittagessen im Deutschen Hause statt, und kurz nach $4 \mathrm{Uhr}$ eröffnete Herr Sch a low mit der folgenden Ansprache die Hauptsitzung:

"Ich eröffne die 60. Jahresversammlung unserer Gesellschaft. Mit besonderer Freude begrüfse ich es, dafs unsere diesjährige Tagung hier in Eberswalde abgehalten wird, in einer Stadt, in welcher ein Mann, dessen Name mit der Entwicklung der Vogelkunde in Deutschland immer verknüpft sein wird, lange gelebt und gewirkt hat: Bernard Altum. In Jahre 1869 als Nachfolger des bekannten Forstentomologen Ratzeburg hierher berufen, hat er bis zu seinem im Jahre 1900 erfolgten Tode als Lehrer der Zoologie an der hiesigen Forstakademie eine segensreiche Tätigkeit entfaltet. Ehe Aituu nach Eberswalde kam, hatte er sich vornehmlich mit der deutschen Vogelkunde beschäftigt. Nach seiner Übersiedelung hierher trat die Ornithologie in den Hintergrund. Zunächst waren es entomologische, bezw. forstentomologische Arbeiten, die seinen Studien Ziel und Richtung gaben. Kaum aber safs er hierin einigermafsen im Sattel, als auch die Beschättigung mit der alten Lieblingswissenschaft wieder in den Vordergrund trat. Durch den Verkehr mit den Männern der grünen Farbe angeregt und im Mittelpunkt eines forstlichzoologischen Versuchswesens stehend, wurden es vornehmlich biologische Fragen, die ihn mehr und mehr beschäftigten und für deren Lösung er Material herbeizuschaffen bemüht war. So enthält denn auch der hier in Eberswalde geschriebene, die Vögel behandelnde Teil seiner „Forstzoologie“, eine Summe wertvoller Beobachtungen, die leider von den Fachgelehrten wenig benutzt worden sind. 
Als Altum seine vogelkundlichen Studien begann, stand er ganz im Banne Naumann'scher Richtung. Voller Begeisterung blickte er zu dem schlichten Autodidakten empor, dessen zwölfbändiges Werk über die Vögel Deutschlands soeben erschienen war, und dessen phänomenale Beobachtungsgabe uns Deutschen die erste, noch heute unübertroffene Beschreibung des äufseren Lebens unserer Vögel, aus eigener Kraft, geschaffen hatte. Naumann war kein spekulativer Kopf. Er schilderte das, was er mit scbarfen Sinnen in der freien Natur beobachtet hatte. Anders Altum. In dialektischer Methode gebildet und zu scharfem Denken in dem Priesterseminar zu Münster erzogen, empfand er, bei aller Verehrung und Anerkennung für Naumanns Arbeit, sehr bald, dafs es eine dringende Forderung sein müsse, der bisher allein geltenden Naturbeschreibung eine Naturer$\mathrm{kl}$ ä r u n g an die Seite zu setzen. In seinem bekannten Tendenzwerk "Der Vogel und sein Leben" suchte er die Fragen zu formulieren und nach dem geringen, vorläufigen Material Schlüsse psychologischer Richtung zu gewinnen. Er stand hierbei in direktem Gegensatz zu Alfred Brehm. Friedrich von Lucanus hat die Anschauungen der beiden Männer, die sich übrigens im Leben freundschaftlich gegenüberstanden, trefflich charakterisiert. Brehm „der glühende Phantast, nach dessen Auffassung dem Tiere weiter nichts fehlt als die Sprache, um auch geistig mit dem Menschen sich messen zu können, Altum der nüchterne, aber auch nicht vorurteilsfreie Forscher und Priester, der im Tier nur eine seelenlose Reflexmaschine erblickt". Wenn Altum in der Beurteilung der Tierseele nach unserer heutigen Auffassung $\mathrm{zu}$ weit ging und viele seiner Schlüsse $\mathrm{zu}$ verwerfen sind, so können wir doch rückbaltlos zugeben, dafs er es gewesen, der zuerst das Dunkel dieser schwierigen Materie zu lichten versucht hat. Die von ihm hierauf bezüglicben Mitteilungen sind nicht nur wegen der gewonnenen Ergebnisse von Wert, sundern sie weisen auch den Weg für die weitere Arbeit. Sie bilden die Brücke $z u$ den heutigen intensiven Untersuchungen zur Psychologie der Vögel, an denen sich auch Mitglieder unserer Gesellschaft mit Erfolg beteiligt baben.

Aber auf einen Punkt möchte ich noch hinweisen. Es will mir scheinen, als ob die Tierpsychologen mit einer gewissen Geringschätzung auf die Vertreter der übrigen Richtungen in der Erforschung des Vogellebens herabsehen. Ich halte dies für bedauerlich und möchte vor einer solchen Auffassung warnen. Nicht allein die Psychologie, nein Morphologie und Verbreitung, Systematik, Nomenklatur und Biologie der äufseren Daseinserscheinungen sind miteinander berufen, die Kenntnis von Leben, Anatomie und Wesen des Vogels in weitesten Sinne zu klären und die Vogelkunde zu fördern."

Nach diesen einleitenden Worten des Vorsitzenden berichten die Herren $\mathrm{Voigt}$ und $\mathrm{H}$ es $\mathrm{s}$ über die Kassenführung und 
beantragen, Herrn Ded iti u s mit bestem Dank die Entlastung $z u$ erteilen. Die Entlastung wird von der Versammlung erteilt, una der Vorsitzende dankt Herrn Deditius für seine bewährte Rechnungsführung und den Revisoren für ihre Mühewaltung.

Herr G r a f v. Z e d l it $z$ berichtet über seine Reise in der Sinai-Halbinsel. Letztere ist bisher noch verhältnismäfsig wenig ornithologisch erforscht worden. 1898 hatte Professor K ö $\mathrm{n}$ i gBonn eine Reise dorthin gemacht, seine Sammlung ist aber noch nicht bearbeitet. Der Ausgangspunkt der Reise des Vortragenden war El-Tor. Von dort aus ging es dem Gebirge zu, das dann von Westen nach Osten durchquert wurde. Ein ausfübrlicher Bericht wird hierüber seitens des Vortragenden veröffentlicht werden, hier seien nur einige kurze zusammenfassende Mitteilungen wiedergegeben. Als Charaktervögel können Cercomela, Pycnonotus, Ammoperdix und eine der Columba schimperi nahestehende Form angesehen werden. In den Tamarisken haust Sylvia rüppclli, im dürren Weidengebüsch treibt Scotocerca inquieta ihr Wesen, von der schon im April flügge Junge gefunden wurden, und Phylloscopus bonelli orientalis haust im niederen Gebüsch. Besonders auffallend ist der schwarze Star Amydrus tristrami; er ähnelt in seinem Verhalten der Alpendohle und belebt mit seinem hellen Pfiff die steilen Felswände, ferner kamen Bubo ascalaphus, Lanius aucheri, Kiparia rupestris obsoleta, Dromolaea leucopyga zur Beobachtung. Carpodacus synoica lebt selbst zur Paarungszeit in kleinen Trupps zusammen, Ammomanes deserti fratercula, Corvus affinis sowie Gypaetus wurden angetroffen, letzterer konnte aber leider nicht erlegt werden. Phoenicurus nesoleuca ist vielleicht. Brutvogel dieser Gebiete, wenigsten wurde er im Frühling angetroffen. Aufserdem wurden sehr viele Zugvögel beobachtet, am ersten Mai sogar 1200 Störche auf einmal.

Im wesentlichen kann gesagt werden, dafs die Vogelwelt der Sinaibalbinsel asiatisch und mit Palästina eng verwandt ist, mit Ägypten hat sie nichts Gemeinsames. Das Gebiet stellt eine bevorzugte Zugstralse dar, die Vögel wandern ganz niedrig in den Tälern entlang, da es dort viel windstiller als auf den Höhen ist. Namentlich unsere heimischen Grasmücken, Sylvia curruca und atricapilla, letztere auch zum Frühjahr hin häufig auch im männlichen Gescblecht mit brauner Kopfplatte, wurden in Mengen beobachtet.

Der Vorsitzende dankt dem Redner für seine anregenden Ausführungen, an die sich ein längerer Meinungsaustausch schliefst.

Herr $\mathrm{H}$ e in r o th ist der Ansicht, dafs es sich bei den auf der Sinaihalbinsel beobachteten Stücken unserer heimischen Zugvogelarten, mit Ausnahme der Störche, wohl nicht um deutsche und westeuropäische Vögel handeln könne, da diese ja doch vorwiegend westlich und südlich ziehen, sondern es liegt der Gedanke nahe, dafs diese Tiere aus Westasien und Rufsland stammen. Er glaubt dies besonders deshalb annehmen zu dürfen, dafs Herr 
Graf v. Zed litz in der Frühjabrszugzeit männliche Mönchsgrasmücken gesammelt hat, die eine noch nicht ganz schwarze Kopfplatte hatten; unser deutscher Mönch pflegt aber bekauntlich schon in seiner ersten Mauser im Spätsommer die schwarze Kopfplatte zu bekommen. Dahingegen hat Heinroth in der Herbstzugzeit in Stud-Rufsland eine grofse Anzahl von Männchen festgestellt, die eine noch braune Kopfplatte aufwiesen; es scheint also, als wenn die östlichen Stücke von $S$. atricapilla sich in diesem Punkt von den westlichen unterscheiden.

Herr R e i c h e n ow wundert sich, dals nach den Angaben des Herrn Grafen v. Z edlitz der dortige Bülbül schlecht singe, während doch die westafrikanische Form $\boldsymbol{P}$. gabunensis und der Verwandte $\boldsymbol{P}$. caffer als gute Sänger gelten. Was die Zugverhältnisse anbetrifft, so sei es wichtig, auch im Herbst im Sinai darauf zu achten: es wäre ja nicht unmöglich, dafs unsere dentschen Vögel auf einem andern Wege in die Heimat zurückzögen als der ist, den sie im Herbst einschlagen.

Herr Eckste in glaubt auch nicht, dals mit Ausnahme der Störche deutsche Vögel durch die Sinaihalbinsel ziehen. Auch die Wachteln zögen wohl über das Mittelmeer und nicht an der syrischen Küste entlang.

Herr Graf $\mathrm{Z}$ e dilitz bemerkt hierzu, dafs er sich keine feste Ansicht darüber bilden könne, ob die im Sinai beobachteten Sylvien west- oder osteuropäische Stücke sind. Was die Wachteln angeht, so hat er beobachtet; dafs diese Vögel im östlichen Nordafrika im Winter viel häufiger sind, als im westlichen, dies schliefst aber nicht aus, dals die ägyptischen Wintergäste aus Osteuropa stammen. $\mathrm{Zu}$ einer Bemerkung des Herrn $\mathrm{H}$ e in $\mathrm{r}$ ot h gibt er noch an, dals er von Sylvia atricapilla und S. curruca im Sinai nie einen Ton gehört habe. $\mathrm{Zu}$ dem Storcbzuge bemerkt Herr $\mathrm{K} \mathrm{r}$ a u s e, dals Ciconia ciconia in Schlesien durchaus nicht dem Laufe der Oder folge, sondern stets quer über den $1600 \mathrm{~m}$ hohen Gebirgskamm ziehe. Herr $\mathrm{N}$ e u m an $\mathrm{n}$ betont, dafs es wohl sehr schwer sei, an einzelnen Vögeln, ohne dafs die Tiere Ringe haben, die Zugstrafse festzustellen. Er ist der Ansicht, dafs die meisten unserer heimischen Vögel nach Westen über ihre alte Urheimat hinwegreisen. Männliche Mönchsgrasmücken mit rotem Oberkopf hat er im Winter auch in Ostafrika beobachtet. Diese kommen, wie Herr K. $\mathrm{N}$ e u $\mathrm{nz}$ i g bemerkt, auch gelegentlich bei uns in Deutschland vor.

Herr v. L u c a n u s fragt, wie hoch etwa die Felswände, an denen man Amydrus und Gypaetus beobachtet, seien, und Herr Graf v. Z ed litz erwidert, dal's man ersteren noch gut auf $100-150 \mathrm{~m}$ sehen könne, von letzterem hat er ein Paar beobachtet, dafs in einer etwa $400 \mathrm{~m}$ hohen Felswand seinen Horst in etwa $250 \mathrm{~m}$ Höhe hatte. Was die Frage nach der Urheimat unserer Zugvögel angeht, so bemerkt Herr D o m e i e r, dafs der 
Storch früher in der Ost-Sahara gelebt habe, und Herr Neumann ptlichtet dieser Ansicht bei.

Herr S c h a low fügt zu dem Vortrage des Herrn G $\mathrm{r}$ a f e $\mathrm{n}$ v. Z e d I it z ergänzend hinzu, dals $P 1$ a te an der Küste des roten Meeres Pandion haliaëtus auf der Erde brütend beobachtet habe.

Herr v. L u can u s hält hierauf seinen mit allseitigem Interesse aufgenommenen Vortrag: „Aeronautische Experimente über die Bestimmung der Höhe fliegender Vögel." Der Inhalt dieses Vortrags ist auszugsweise bereits im Bericht über die Aprilsitzung 1911 der Deutschen Ornithologischen Gesellschaft im Journ. f. Orn. wiedergegeben worden, und der Vortragende hat die Absicht, in Kürze seine Beobachtungen selbst ausführlich zu veröffentlichen. Es sei hier nur noch bemerkt, dals Herr v. $L$ u c a n u s sicb in Ergänzung der Ballonversucbe die dabei untersuchten Flugbilder in $1 / 10$ natürlicher Grölse auf grofse weifse Papierbogen verkleinert hat und dabei feststellen konnte, dafs sich die Erkennbarkeit dieser Objekte unter einander genau so verhält, wie die der wirklichen bezw. ausgestopften Vögel. Mit Hilfe dieses Mittels kann man bei bekannter Fluggrölse eines Vogels seine Sichtbarkeit ohne weiteres unter Bezugnahme aut die beim Ballonversuch festgestellten Werte berechnen. Aufser seinen eigenen Tabellen bat er noch die Angaben Gätkes und Humboldts über die Grenzen der Sichtbarkeit fliegender Vögel vorgeführt, und es stellte sich dabei heraus, dafs sich diese beiden Beobachter um etwa $3000 \mathrm{~m}$ überschätzt hatten.

Herr $\mathrm{H}$ e in r o th legt eine Anzahl von Luftröhren und Knochentrommeln (Bulla ossea) verschiedener Anatiden-Männchen vor. Er zeigt die Unterschiede dieser Gebilde bei den Plectropterinae, den Gattungen Casarca, Dendrocygna, Lampronessa, Aex, Anas, Nettium, Querquedula, ferner bei den Fuligulinen Netta und Clangula sowie von Merganser serrator. Zum Vergleich bat er von vielen Formen auch die sehr einfachen Luftröhren weiblicher Stücke mitgebracht, die aufser bei Dendrocygna keinerlei Auftreibungen und Knochentrommeln zeigen. Er wies darauf hin, dafs bei vielen Entenmännchen zur Erzeugung eines einzigen Balzlautes ein kompliziertes Organ entstanden sei; ein Umstand, der es aber gleichzeitig mit sich bringt, dafs sie im übrigen vollkommen stumm geworden sind: man hat es also hier so recht eigentlich mit einer Sackgasse zu tun, in die diese Vogelgruppe geraten ist. Das Nähere über diese Betrachtungen findet sich im wesentlichen in der im Bericht über den V. Internationalen Ornithologen-Kongress Berlin 1910 erschienenen Arbeit, „Beiträge zur Biologie, namentlich Ethologie und Psychologie der Anatiden." Der Vortragende bezeichnete es als den Hauptzweck seiner Demonstration, zum Sammeln von Luftröhren und Knochentrommeln anzuregen; leider würden diese Organe so gut wie niemals aufgehoben, sodafs wir über sie bei den seltener vorkommenden Anatiden wie Cyanochen, Pteronetta, Malacorynchus, Stictonetta, 
Tachyeres, Eniconetta, Merganetta, Erismatura, Biziura und vielen andern z. T. noch vollkommen im Unklaren sind, trotzdem sie wichtige Fingerzeige für die systematische Stellung und die nähere Verwandtschaft dieser Formen geben können.

Etwa um 7 Uhr schliefst der Vorsitzende die Sitzung und dankt namens aller Mitglieder den Herren Vortragenden für die interessanten Mitteilungen, sowie Herrn Professor Eckstein fül die Liebenswürdigkeit, mit der er die Führung in seinem Museum übernommen. Herr Eckstein erwidert mit einigen herzlichen Worten.

Der gröfste Teil der Teilnehmer fand sich am nächsten Morgen, den 9. Oktober, wieder zusammen. Man bestieg den Zug nach Nieder-Finow, besichtigte dort bei etwa zweistündigem Aufenthalt die ebenso interessanten als grofsartigen und umfangreichen, im Entstehen begriffenen Schleusenanlagen, fuhr dann gegen Mittag nach Freienwalde weiter und nach einem gemeinsam eingenommenen Mittagessen macbte man sich auf den Weg nach der berrlich gelegenen Besitzung des Herrn Dr. L a valle in Scbiffmüble. Es war ein Genufs, die ansgedehnte Rassegeflügel. zucht dieses Herrn, der die Mitglieder in liebenswürdigster Weise durch alle Räume geleitete, zu bewundern. Mechelner Kuckucks-Sperber, Brahmas, Langshans, Orpingtons, PlymouthRocks, Minorcas und Italiener in mehreren Farbenschlägen, ebenso allerliebste wie typische deutsche Möwchentauben, Strasser und Koburger Lerchen, sowie Emdener Gänse und indische I aufenten waren in grolsen sauberen Ausläufen und Ställen aufs zweckmälsigste untergebracht. Etwa 600 Hühner trieben dort ibr Wesen, und lange Zeit kreiste ein kleiner Trupp fluggewandter Hausenten (Hochtugenten) über Haus und Garten. Eine Einladung des Herrn La va lle zum Kaffee und zu einem Schluck seines aus Hühnereidotter selbst hergestellten Eierkognacs nahmen die Beteiligten mit Freuden an, zumal inzwischen draufsen recht kühles, unfreundliches, regnerisches Wetter eingetreten war. Bald nach 5 Uhr brach man unter herzlichen Dankesworten an Herrn Dr. Lavalle auf, um noch rechtzeitig den nach Berlin fahrenden Zug in Freienwalde zu erreichen. O. Heinroth.

\section{Dentsche Ornithologisehe Gesellschaft. Bericht über die Septembersitzung 19II.}

Verhandelt Berlin, Montag den 4. September, abends 8 Uhr im Architektenvereinshause Wilhelmstrafse 92.

Anwesend die Herren: Freiher $r$ Geyr von Schweppenburg, Grote, Jung, Neunzig, Graf v. Zedlitz u. Trützschler, Deditius, Reichenow, Schalow, Krause, 0 . Neumann und $\mathrm{Hein}$ roth. 